\title{
Phase diagram and dissolution studies of the fenofibrate-acetylsalicylic acid system
}

\author{
Agata Górniak - Alina Wojakowska • \\ Bożena Karolewicz $\cdot$ Janusz Pluta
}

Received: 21 July 2010/ Accepted: 29 October 2010/Published online: 12 December 2010

(C) The Author(s) 2010. This article is published with open access at Springerlink.com

\begin{abstract}
Enhancement of the dissolution rate of poorly soluble compounds through the formation of drug-drug eutectics was investigated using fenofibrate and acetylsalicylic acid. Solid-liquid equilibria in the system under study were investigated by differential scanning calorimetry (DSC). The phase diagram for the whole range of compositions was constructed. In addition, existence of a metastable polymorph of fenofibrate has been confirmed. The investigation has revealed that acetylsalicylic acid and fenofibrate form a simple eutectic mixture containing 0.958 mol fraction of fenofibrate at the eutectic point. Dissolution rate improvement of fenofibrate correlated with the phase diagram. The amount of fenofibrate released from the solid dispersions that contained fenofibrate as the eutectic mixture with acetylsalicylic acid was at least threefold higher compared to untreated fenofibrate.
\end{abstract}

Keywords Acetylsalicylic acid - Dissolution enhancement · DSC · Eutectic · Fenofibrate ·

Phase diagram $\cdot$ Polymorphism $\cdot$ Solubility

\section{Introduction}

Fenofibrate (isopropyl 2-[4-(4-chlorobenzoyl)phenoxy]-2methylpropionate), known for more than 30 years, is a lipid-regulating agent available as tablets and capsules for

\footnotetext{
A. Górniak $(\bowtie) \cdot$ A. Wojakowska

Department of Inorganic Chemistry, Wroclaw Medical

University, Szewska 38, 50-139 Wroclaw, Poland

e-mail: agata.gorniak@chnorg.am.wroc.pl

B. Karolewicz · J. Pluta

Department of Drug Form Technology, Wroclaw Medical

University, Szewska 38, 50-139 Wroclaw, Poland
}

oral administration. Independently, fenofibrate produces antithrombotic and anti-inflammatory effects. Thus, it is used in the treatment of hyperlipidemia, heart disease, and diabetic complications [1].

The chemical structure (Fig. 1) and some physicochemical properties of the fenofibrate were presented by Shoji et al. [2] based on elemental analysis and UV, IR, NMR, XRD, and mass spectra examination as well as on hygroscopicity, solubility, distribution coefficients, and TG and DTA measurements. It has been found that fenofibrate is a sparingly water soluble compound, the volume of water to dissolve one gram of the fenofibrate at $37^{\circ} \mathrm{C}$ being larger than $410 \mathrm{dm}^{3}$. The melting point was determined to be $81.6{ }^{\circ} \mathrm{C}$. No thermal effects besides melting were observed on DTA curves nor any mass loss was noticed during thermogravimetric measurements from 20 to $200{ }^{\circ} \mathrm{C}$ [2].

Subsequently, thermodynamic quantities for melting (melting point: $80.5^{\circ} \mathrm{C}$, enthalpy of melting: $32.4 \mathrm{~kJ} \mathrm{~mol}^{-1}$ ), glass transition temperature $\left(T_{\mathrm{g}}=-20.0^{\circ} \mathrm{C}\right)$, and heat capacity values as a function of temperature for the crystalline and amorphous phase of fenofibrate were obtained by Zhou et al. [3] as a result of differential scanning calorimetry (DSC) and modulated temperature DSC (MTDSC) measurements. Crystallization studies performed by those authors indicated an intermediate tendency for fenofibrate to crystallize from amorphous state.

Two forms of crystalline fenofibrate have been reported. A stable modification crystallizes in the triclinic space group Pī [4]. Its structure consists of alternating arrangements of aliphatic and aromatic layers formed by molecules, which is the consequence of the absence of groups donating hydrogen-bonding interactions. A metastable polymorph of fenofibrate, crystallizing from an amorphous form and melting at $73.5^{\circ} \mathrm{C}$, was discovered by Di Martino 


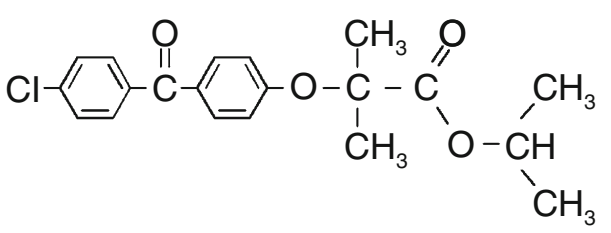

Fig. 1 Chemical structure of fenofibrate (isopropyl 2-[4-(4-chlorobenzoyl)phenoxy]-2-methylpropionate)

et al. [5], based on DSC experiments. It can be formed also on grinding or when stored in a very humid atmosphere. A transition into the stable form is rather slow given that the metastable modification has been still identified in the mixture by XRD after 90 days.

Acetylsalicylic acid (aspirin), which is also a poorly water soluble compound, has been used as an analgesic, antipyretic, and anti-inflammatory nonsteroidal drug (agent) for more than a century. In recent years, however, since the time when it was demonstrated that a low-dose acetylsalicylic acid revealed an inhibiting effect on platelet aggregation, aspirin has been applied to prevent complications of atherosclerotic cardiovascular disease such as myocardial infarction and stroke [1].

Aspirin is stable on heating until it melts at about $140{ }^{\circ} \mathrm{C}$ and then it decomposes [6, 7]. Acetylsalicylic acid is thought to exist in one crystal structure, although some experimental as well as computational attempts were made to found new forms [8]. Nevertheless, it seems that polymorphism of aspirin is not commonly acknowledged at present [9].

The aqueous solubility behavior of a drug is a key determinant of its oral delivery. Poor water solubility and slow dissolution are often the main reasons for the rejection of potentially active drugs [10, 11]. Several approaches have been employed to overcome this problem, such as the preparation of solid dispersions of an active agent in a biologically inert matrix [12-14], drug dissolution in the presence of polymeric micelles in the dissolution medium [15], addition of surfactants [15-18], micronization-particle size reduction to nano- or microsize [19-21], melt granulation technique [22, 23] and formation of eutectics $[24,25]$, including drug-polymer component systems $[26,27]$.

The use of eutectic mixtures for pharmaceutical applications was first described by Sekiguchi and Obi [28]. In the eutectic composition, both components are in reduced particle size and well dispersed. The large surface area of the obtained suspension should result in an enhanced dissolution rate and thereby improved bioavailability. The unique property of an eutectic mixture is that it has a lower melting temperature than that of either of the pure components. For this reason it can be used also as transdermal permeation enhancer [10].
Thermal methods are more and more used in pharmaceutical applications [29, 30], both in drug development and in industrial processes. DSC and related techniques widely contribute to the study of, e.g., the stability of drugs [31-33], their polymorphism [34], and drug-excipient compatibility or chemical interactions [35-37].

The aim of this study was to determine the eutectic composition and temperature for the system fenofibrateacetylsalicylic acid, through the construction of the phase diagram based on the results of DSC heating experiments, as well as to investigate the solubility and dissolution rate of prepared mixtures.

\section{Experimental section}

DSC studies

Fenofibrate and acetylsalicylic acid were commercially available products: $99 \%$, Aldrich. Finely powdered physical mixtures were prepared from accurately weighed quantities of each component, next very thoroughly ground together for at least 5 min using an agate mortar and pestle. Samples were weighed using a Mettler Toledo AT 261 microbalance $( \pm 0.01 \mathrm{mg})$. The total mass of a sample was between 4 and $10 \mathrm{mg}$. Twenty-five samples of acetylsalicylic acid + fenofibrate binary mixtures with following mole fractions of fenofibrate $\left(x_{\mathrm{F}}\right)$ : $0.950,0.900,0.850$, $0.800,0.750,0.700,0.651,0.603,0.550,0.531,0.498$, $0.445,0.401,0.350,0.310,0.253,0.200,0.150,0.099$, $0.070,0.060,0.050,0.040,0.029$, and 0.020 were prepared.

The DSC curves of each mixture were obtained using a Mettler Toledo DSC 25 measuring cell, equipped with a heat flow sensor and joined via interface TA Controller TC 15 to a computer. Measurements were driven by STAR $^{\mathrm{e}}$ software.

Samples for DSC measurements were sealed in $40 \mu \mathrm{L}$ standard aluminum crucibles with a single hole punched in the lid. An empty pan of the same type was employed as a reference. The DSC instrument was calibrated using the melting point of indium (156.6 \pm 0.3$)$ as a standard [38]. DSC scans of each mixture were performed at a heating rate of $5^{\circ} \mathrm{C} \mathrm{min}{ }^{-1}$ in the temperature range from 30 to $140{ }^{\circ} \mathrm{C}$. For pure acetylsalicylic acid DSC measurements were taken in the temperature range from 30 to $160{ }^{\circ} \mathrm{C}$. The DSC cell was purged with a stream of dry argon at a rate of $50 \mathrm{~cm}^{3} \mathrm{~min}^{-1}$.

Dissolution studies

Dissolution tests of pure drugs and their physical mixtures were determined on an apparatus 2 (paddle) [39]. Tablets were prepared without addition of excipients. Compression 
was carried out with the use of Erweka Tablet Press. Press force $8 \mathrm{kN}$ was used. The paddle rotation speed was $100 \mathrm{rpm}$. The temperature was kept at $37^{\circ} \mathrm{C}$ and the volume of the dissolution medium was $1000 \mathrm{~cm}^{3}$. Experiments were performed in a $0.1 \mathrm{~mol} \mathrm{dm}^{-3}$ sodium lauryl sulfate water solution. Samples were passed through $35-\mu \mathrm{m}$ filters. The concentration of fenofibrate and acetylsalicylic acid in solution was measured spectrophotometrically (Jasco V650 Spectrophotometer) at a wavelength of 291 and $275 \mathrm{~nm}$ for fenofibrate and acetylsalicylic acid, respectively. Concentration of dissolved drugs was calculated by reference to predetermined standard curves. Linear calibration curves were obtained between 0.02 and $5 \mathrm{mg} /$ $\mathrm{dm}^{3}$ for fenofibrate and between 1 and $100 \mathrm{mg} / \mathrm{dm}^{3}$ for acetylsalicylic acid.

\section{Results and discussion}

Thermograms of pure acetylsalicylic acid, obtained at a heating rate of $5{ }^{\circ} \mathrm{C} \mathrm{min}^{-1}$ in the temperature range from 30 to $160{ }^{\circ} \mathrm{C}$, show only one endothermal effect $\left(\Delta_{\text {fus }} H=\right.$ $25.9 \mathrm{~kJ} \mathrm{~mol}^{-1}$ ), corresponding to melting at $141.9{ }^{\circ} \mathrm{C}$, which confirms that under these conditions the substance is stable and does not decompose.

Our examinations confirmed the existence of a metastable polymorphic form of fenofibrate [5]. This is illustrated in

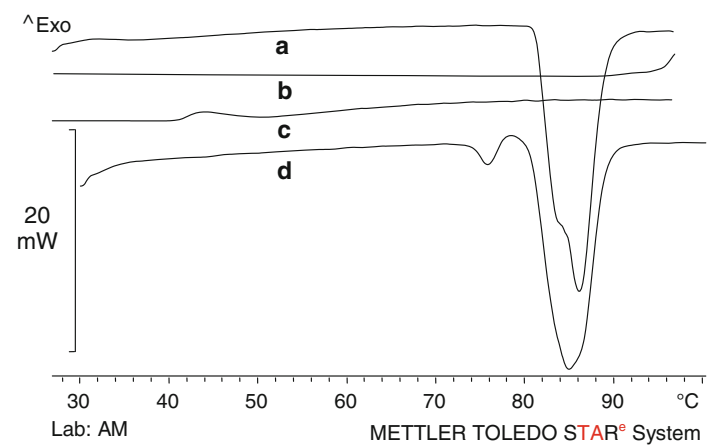

Fig. 2 Differential scanning calorimetry (DSC) study of fenofibrate: (a) first heating run, $(b)$ cooling to room temperature, $(c)$ second heating immediately after cooling, and $(d)$ heating following after 45 days of storage. The all runs were performed at $10{ }^{\circ} \mathrm{C} \mathrm{min}-1$
Fig. 2 by four DSC experiments. Only the melting of the stable form I is observed during the first heating of the fenofibrate powder (Fig. 2a). However, the shape of the melting endotherm looks as it comprised overlapping effects. Cooling after melting the sample with a rate of $10{ }^{\circ} \mathrm{C} \mathrm{min}^{-1}$ causes the solidification to the amorphous form of the drug (Fig. 2b). During the second heating a broad exothermic peak was observed (Fig. 2c) which revealed crystallization from the amorphous phase. The last DSC curve (Fig. 2d) presents heating of fenofibrate after 45 days of storage at room temperature, showing the melting endotherm of a metastable form II followed by the exothermic crystallization peak and the endothermic melting peak of the stable form I. The metastable form II did not appear after grinding and compressing process. The experimentally determined values of melting points $\left(T_{\text {fus }}\right)$ and melting enthalpy $\left(\Delta_{\text {fus }} H\right)$ for both polymorphs and the temperature range of crystallization from amorphous form $\left(T_{\mathrm{c}}\right)$ have been gathered in Table 1 and compared with the literature data.

Figure 3 shows the phase diagram of the investigated system constructed on the basis of the DSC results. The DSC curves of the various mixtures clearly indicated that the investigated compounds formed a binary eutectic system. The first peak consistently appeared near $76^{\circ} \mathrm{C}$ (Fig. 4), indicating the eutectic reaction. This temperature is also



Fig. 3 Phase equilibrium diagram of the investigated system acetylsalicylic acid $(A)$-fenofibrate $(F)$

Table 1 Crystallization from amorphous form and thermodynamic quantities of crystalline forms of fenofibrate

\begin{tabular}{|c|c|c|c|c|c|c|}
\hline \multirow[t]{2}{*}{ Method } & \multirow{2}{*}{$\begin{array}{l}\text { Amorphous form } \\
T_{\mathrm{C}} /{ }^{\circ} \mathrm{C}\end{array}$} & \multicolumn{2}{|c|}{ Metastable form II } & \multicolumn{2}{|c|}{ Stable form I } & \multirow[t]{2}{*}{ Ref. } \\
\hline & & $T_{\text {fus }} /{ }^{\circ} \mathrm{C}$ & $\Delta_{\text {fus }} H / \mathrm{kJ} \mathrm{mol}^{-1}$ & $T_{\text {fus }} /{ }^{\circ} \mathrm{C}$ & $\Delta_{\text {fus }} H / \mathrm{kJ} \mathrm{mol}^{-1}$ & \\
\hline DTA & - & - & - & 81.6 & - & {$[2]$} \\
\hline DSC & $45-60$ & 73.5 & $10.3 \pm 6.3$ & 80.2 & $33.2 \pm 3.5$ & {$[5]$} \\
\hline DSC & 40 & - & - & 80.5 & 32.4 & {$[3]$} \\
\hline DSC & $34-56$ & 74.0 & 0.9 & 81.4 & 27.3 & [This work] \\
\hline
\end{tabular}




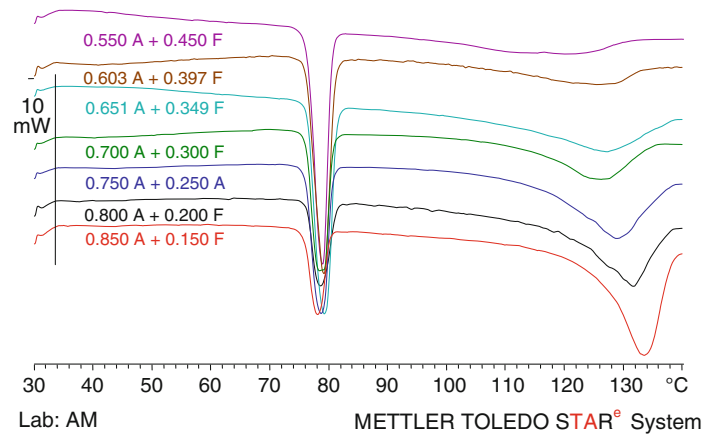

Fig. 4 Examples of DSC curves showing both eutectic and liquidus events in the range of compositions $x_{\mathrm{A}}>x_{\mathrm{F}}$

known as the temperature of solidus on a phase diagram. The second peak (temperature of liquidus) was generally wider, indicating that complete melting took place over a temperature range. The DSC scans are typical of eutectic systems. Only these two kinds of thermal effects are shown by DSC curves for the whole range of compositions. The melting points of the pure components (acetylsalicylic acid: $141.9^{\circ} \mathrm{C}$, fenofibrate: $81.4^{\circ} \mathrm{C}$ ) were depressed due to the existence of the other component in the mixture as shown in the phase diagram in Fig. 3. The examples of DSC curves showing both eutectic and liquidus events are given in Fig. 4. It should be added that DSC curves of pure acetylsalicylic acid as well as those of pure fenofibrate have shown sharp melting endotherms which permitted to determine the respective melting points precisely.

The onset of the first peak occurs approximately at the same temperature $76.2{ }^{\circ} \mathrm{C}$ for all the compositions and represents the eutectic reaction:

Solid acetylsalicylic acid (A) + solid fenofibrate (F) $=$ liquid $(\mathrm{L})$.

For this type of transition the peak position does not vary with the content of a component, whereas the heat of the thermal events rises when approaching to the eutectic point. In this study the thermal effect corresponding to the eutectic reaction was noticeable on DSC curves for all the mixtures examined (Figs. 4, 5, and 6).

The second event corresponding to the thermal effect of liquidus gradually diminished with increasing mole fraction of fenofibrate and was no longer noticed around $x_{\mathrm{F}}=0.750$. The DSC scans recorded for $x_{\mathrm{F}} \geq 0.750$ showed the characteristic overlap of two endotherms (eutectic and liquidus events) into a single peak near the eutectic temperature (Fig. 6).

Values of the eutectic melting enthalpy $\Delta H / \mathrm{kJ} \mathrm{mol}^{-1}$ for a given mixture, determined by integration of the eutectic peak area on DSC curves, are plotted in Fig. 7 versus mole fraction of fenofibrate $x_{\mathrm{F}}$ (Tamman's triangle construction [40]). The maximal enthalpy value has been found for

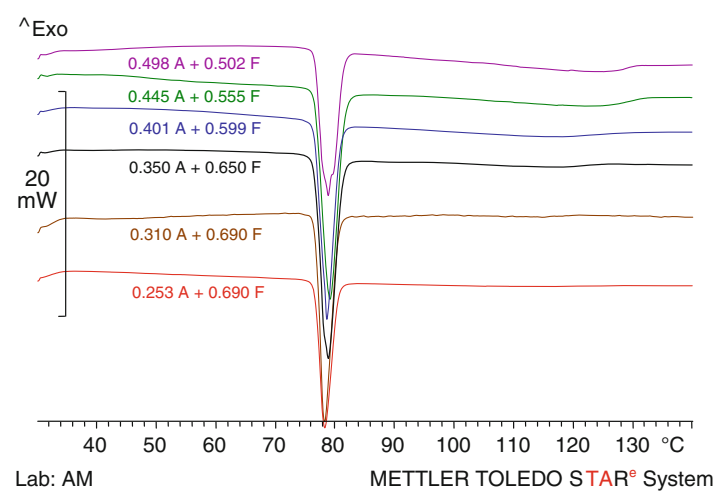

Fig. 5 Examples of DSC curves performed at a heating rate of $5{ }^{\circ} \mathrm{C} \mathrm{min}{ }^{-1}$ showing a gradual disappearance of the thermal effect of liquidus with increasing mole fraction of fenofibrate before the eutectic point

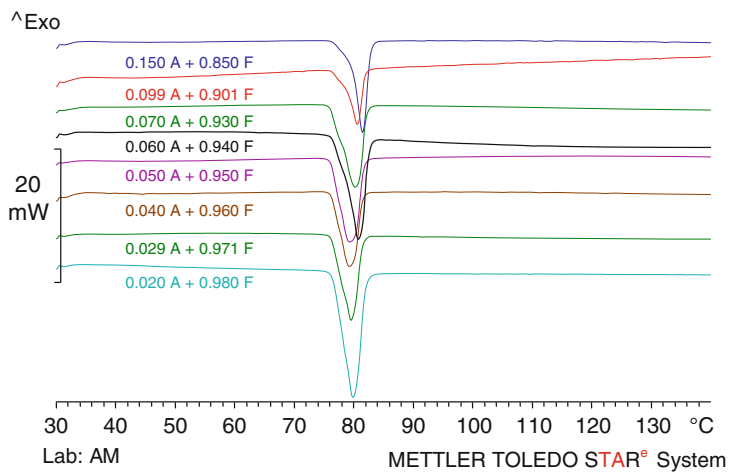

Fig. 6 Examples of DSC curves performed at a heating rate of $5{ }^{\circ} \mathrm{C} \mathrm{min}-1$ and showing overlap of two endotherms (eutectic and liquidus event) into a single peak near the eutectic temperature

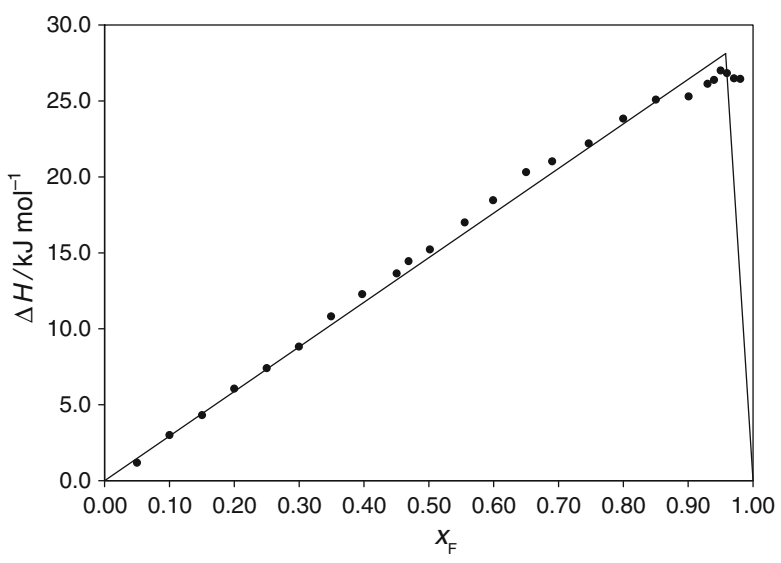

Fig. 7 Tamman's triangle construction for the eutectic melting enthalpy $\Delta H$ at $76.2^{\circ} \mathrm{C}$

$x_{\mathrm{F}}=0.958$ which corresponds to the composition of the eutectic point and is in agreement with the phase diagram (Fig. 3). Thermal effect of the eutectic transition goes to zero for compositions corresponding to pure components. 


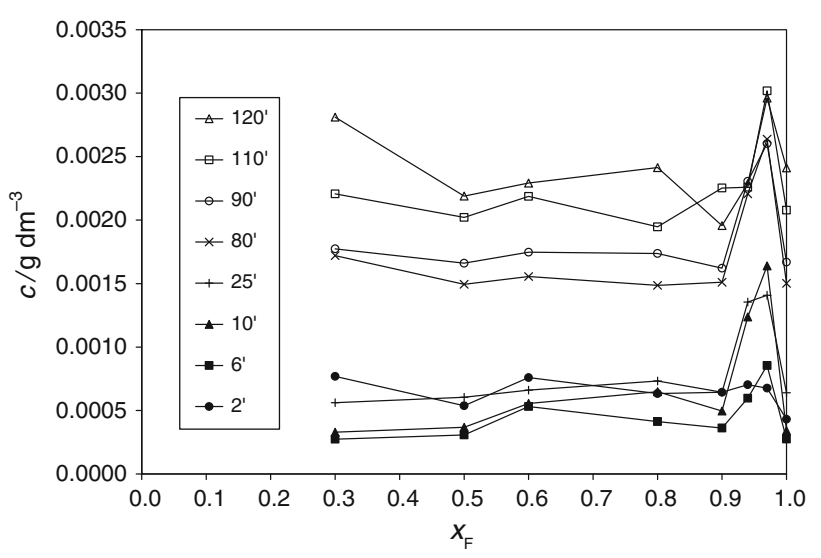

Fig. 8 Concentration $c$ of dissolved fenofibrate versus mole fraction of the drug in a solid mixture $\left(x_{\mathrm{F}}\right)$ in 2 (filled circle), 6 (filled square), 10 (filled triangle), 25 (plus), 80 (times), 90 (open circle), 110 (open square), and 120 (open triangle) minutes of dissolution test

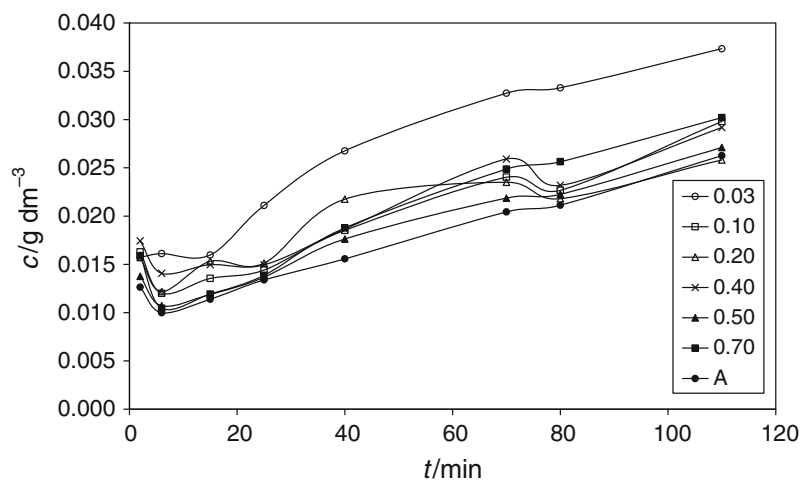

Fig. 9 Concentration $c$ of acetylsalicylic acid in $0.1 \mathrm{~mol} \mathrm{dm}^{-3}$ sodium lauryl sulfate water solution versus time $t$ for pure acetylsalicylic acid (A) and mixtures containing 0.03 (open circle), 0.10 (open square), 0.20 (open triangle), 0.40 (times), 0.50 (filled triangle), and 0.70 (filled square) mole fraction of acetylsalicylic acid

This confirms that there is no mutual miscibility in the solid state and no formation of terminal solid solutions at either side of the phase diagram. Finally, parameters of the eutectic point have been established as follows:

Composition: mole fraction of fenofibrate 0.958; mole fraction of acetylsalicylic acid 0.042

Temperature: $76.2^{\circ} \mathrm{C}$.

Use of the investigated phase diagram in selecting the optimum ratio of components to enhance dissolution rates of these drugs is possible.

The results of dissolution studies presented in Fig. 8 compare solid dispersions for the whole range of compositions. The most characteristic feature is a noticeable increase in dissolution of the low-soluble fenofibrate observed for the eutectic region. The factor of increase in the fenofibrate dissolution for the eutectic region in comparison to that for the pure substance was $\sim 3$ for a mechanical (physical) mixture, followed by thorough grinding in a mortar. Values of the acetylsalicylic acid concentration versus dissolution time for various acetylsalicylic acid-fenofibrate mixtures are plotted in Fig. 9. Dissolution studies of acetylsalicylic acid also showed a characteristic (although not so significant) increase at the eutectic region.

\section{Conclusions}

This investigation illustrates the potential use of eutectic mixtures of fenofibrate with acetylsalicylic acid for the preparation of new double drug combination formulations with improved bioavailability. The poor water soluble drug acetylsalicylic acid and practically insoluble compound fenofibrate form a simple eutectic system containing $0.958 \mathrm{~mol}$ fraction of fenofibrate at the eutectic point. The most characteristic feature is a noticeable increase in the rate of dissolution of fenofibrate observed for the eutectic formation region.

Due to the limited solubility of fenofibrate in water, the melting point depression effect, through eutectic formation with acetylsalicylic acid using simple mechanical activation (grinding method), provides an attractive alternative for the preparation of formulations (granules, tablets, and capsules) with potential increased oral bioavailability.

Open Access This article is distributed under the terms of the Creative Commons Attribution Noncommercial License which permits any noncommercial use, distribution, and reproduction in any medium, provided the original author(s) and source are credited.

\section{References}

1. Brunton LL, Lazo JS, Parker KL, editors. Goodman \& Gilman's the pharmacological basis of therapeutics. 11th ed. New York: McGraw-Hill Companies; 2006.

2. Shoji R, Watanabe T, Tashiro S, Shi S. Chemical structure and physico-chemical properties of fenofibrate. Iyakuhin Kenkyu. 1995;26:386-97.

3. Zhou D, Zhang GGZ, Law D, Grant DJW, Schmitt EA. Physical stability of amorphous pharmaceuticals: importance of configurational thermodynamic quantities and molecular mobility. J Pharm Sci. 2002;91:1863-72.

4. Henry RF, Zhang GZ, Gao Y, Buckner IS. Fenofibrate. Acta Crystallogr E. 2003;59:0699-700.

5. Di Martino P, Palmieri GF, Martelli S. Evidence of a metastable form of fenofibrate. Pharmazie. 2000;55:625-6.

6. Ribeiro YA, Caires ACF, Boralle N, Ionashiro M. Thermal decomposition of acetylsalicylic acid (aspirin). Thermochim Acta. 1996;279:177-81.

7. Long GT, Vyazovkin S, Gamble N, Wight CA. Hard to swallow dry: kinetics and mechanism of the anhydrous thermal decomposition of acetylsalicylic acid. J Pharm Sci. 2002;91:800-9.

8. Payne RS, Rowe RC, Roberts RJ, Charlton MH. Potential polymorphs of aspirin. J Comput Chem. 1999;20:262-73. 
9. Bond AD, Boese R, Desiraju GR. On the polymorphism of aspirin. Angew Chem Int Ed. 2007;46:615-7.

10. Riga AT, Oberoi LM, Alexander KS. Differential scanning calorimetry as a preformulation tool for studying eutectics relevant in pharmaceuticals. Am Pharm Rev. 2004;7:18-23.

11. Avula SG, Alexander K, Riga A. Predicting eutectic behavior of drugs and excipients by unique calculations. J Therm Anal Calorim. 2010;99:655-8.

12. Sheu M-T, Yeh C-M, Sokoloski TD. Characterization and dissolution of fenofibrate solid dispersion systems. Int J Pharm. 1994;103:137-46.

13. Palmieri GF, Antonini I, Martelli S. Characterization and dissolution studies of PEG4000/fenofibrate solid dispersions. STP Pharma Sci. 1996;6:188-94.

14. Kanaze FI, Kokkalou E, Niopas I, Georgarakis M, Stergiou A, Bikiaris D. Thermal analysis study of flavonoid solid dispersions having enhanced solubility. J Therm Anal Calorim. 2006;83: 283-90.

15. Granero GE, Ramachandran Ch, Amidon GL. Dissolution and solubility behavior of fenofibrate in sodium lauryl sulfate solutions. Drug Dev Ind Pharm. 2005;31:917-22.

16. Sant VP, Smith D, Leroux J-Ch. Enhancement of oral bioavailability of poorly water-soluble drugs by poly(ethylene glycol)block-poly(alkyl acrylate-co-methacrylic acid) self-assemblies. J Control Release. 2005;104:289-300.

17. Sant VP, Smith D, Leroux J-Ch. Novel pH-sensitive supramolecular assemblies for oral delivery of poorly water soluble drugs: preparation and characterization. J Control Release. 2004;97: 301-12.

18. Temeljotov FD, Mohar M, Kofler B, Kotnik S, Resman A. Solubilization and dissolution enhancement for sparingly soluble fenofibrate. Acta Pharm. 1996;46:131-6.

19. Bettini R, Rossi A, Lavezzini E, Frigo E, Pasquali I, Giordano F. Thermal and morphological characterisation of micronized acetylsalicylic acid powders prepared by rapid expansion of a supercritical solution. J Therm Anal Calorim. 2003;73:487-97.

20. Su C-S, Tang M, Chen Y-P. Micronization of nabumetone using the rapid expansion of supercritical solution (RESS) process. J Supercrit Fluids. 2009;50:69-76.

21. Papageorgiou GZ, Docoslis A, Georgarakis M, Bikiaris D. The effect of physical state on the drug dissolution rate: miscibility studies of nimodipine with PVP. J Therm Anal Calorim. 2009;95:903-15.

22. Passerini N, Albertini B, González-Rodríguez ML, Cavallari C, Rodriguez L. Preparation and characterization of ibuprofen-poloxamer 188 granules obtained by melt granulation. Eur J Pharm Sci. 2002;15:71-8.

23. Yadav VB, Yadav AV. Enhancement of solubility and dissolution rate of fenofibrate by melt granulation technique. Int $\mathrm{J}$ PharmTech Res. 2009;1:256-63.

24. Liu D, Fei X, Wang S, Jiang T, Su D. Increasing solubility and dissolution rate of drugs via eutectic mixtures: itraconazole-poloxamer 188 system. Asian J Pharm Sci. 2006;1:213-21.
25. Pasquali I, Bettini R, Giordano F. Thermal behaviour of diclofenac, diclofenac sodium and sodium bicarbonate compositions. J Therm Anal Calorim. 2007;90:903-7.

26. Law D, Wang W, Schmitt EA, Qiu Y, Krill SL, Fort JJ. Properties of rapidly dissolving eutectic mixtures of poly(ethylene glycol) and fenofibrate: the eutectic microstructure. J Pharm Sci. 2003; 92:505-15.

27. Vippagunta SR, Wang Z, Hornung S, Krill SL. Factors affecting the formation of eutectic solid dispersions and their dissolution behaviour. J Pharm Sci. 2007;96:294-304.

28. Sekiguchi K, Obi N. Studies on absorption of eutectic mixture. I. A Comparison of the behavior of eutectic mixture of sulfathiazole and that of ordinary in man. Chem Pharm Bull. 1961;9:866-72.

29. Thompson KC. Pharmaceutical applications of calorimetric measurements in the new millennium. Thermochim Acta. 2000; 355:83-7.

30. Canotilho J, Castro RAE, Rosado MTS, Nunes SCC, Cruz MSC, Redinha JS. Thermal analysis and crystallization from melts: $\beta$ adrenergic compounds. J Therm Anal Calorim. 2010;100:423-9.

31. Wesolowski M, Szynkaruk P. Thermal decomposition of methylxanthines: interpretation of the results by PCA. J Therm Anal Calorim. 2008;93:739-46.

32. Mielcarek J, Naskrent M, Grobelny P. Photochemical properties of simvastatin and lovastatin induced by radiation. $\mathrm{J}$ Therm Anal Calorim. 2009;96:301-5.

33. Yoshihashi Y, Yonemochi E, Maeda Y, Terada K. Prediction of the induction period of crystallization of naproxen in solid dispersion using differential scanning calorimetry. J Therm Anal Calorim. 2010;99:15-9.

34. Bruni G, Milanese C, Berbenni V, Sartor F, Villa M, Marini A. Crystalline and amorphous phases of a new drug. J Therm Anal Calorim. 2010;102:297-303.

35. Santos AFO, Basílio ID Jr, de Souza FS, Medeiros AFD, Pinto MF, de Santana DP, Macêdo RO. Application of thermal analysis of binary mixtures with metformin. J Therm Anal Calorim. 2008;93:361-4.

36. Bernardi LS, Oliveira PR, Murakami FS, Silva MAS, Borgmann SHM, Cardoso SG. Characterization of venlafaxine hydrochloride and compatibility studies with pharmaceutical excipients. J Therm Anal Calorim. 2009;97:729-33.

37. Bruni G, Berbenni V, Milanese C, Girella A, Marini A. Drugexcipient compatibility studies in binary and ternary mixtures by physico-chemical techniques. J Therm Anal Calorim. 2010;102: 193-201.

38. Höhne GWH, Hemmninger W, Flammersheim H-J. Differential scanning calorimetry. Berlin: Springer; 1996.

39. United States Pharmacopoeia. USP 32 NF 27. 2009.

40. Campanella L, Micieli V, Tomassetti M, Vecchio S. Solid-liquid phase diagrams of binary mixtures. Acetylsalicylic acid(1)+ $\mathrm{E}(2)(\mathrm{E}=$ salicylic acid, polyethylene glycol 4000, D-mannitol). J Therm Anal Calorim. 2010;99:887-92. 